\title{
Benefits of Video Presentations in Product Design
}

\author{
Alex Lobos \\ Assistant Professor of Industrial Design, College of Imaging Arts and Sciences, \\ Rochester Institute of Technology, USA
}

\begin{abstract}
Product Design uses a human-centered process to develop solutions that solve unmet user needs. Because of the sequential nature of this activity, final designs are often presented in printed process books or digital slideshows, which visually communicate the development of the solution from start to finish rather than focusing just on the final result. Storytelling is a key element to consider when creating these process books in order to communicate the design solution as well as where it came from. An alternative to these presentations is the use of short videos, which offer the advantage of communicating the design process with a richer narrative and engaging style. The use of videos for final presentations both in the classroom and in professional practice offers a wider array of opportunities for effective storytelling of design projects while reaching a broader audience.
\end{abstract}

\section{Introduction}

Product Design creates solutions for users unmet needs by applying a process that goes from assimilation of the user's context to investigating problems and developing potential solutions, all leading to design solutions that are communicated to stakeholders in hopes of implementation [1]. When presenting product design projects, showing the process and decision-making is just as important as the final solution itself. Traditional formats for presenting this type of work involve the creation of process books, which can be printed or displayed digitally. These formats are widely used and accepted by the industry but the use of more dynamic media such as video is becoming a popular alternative for presenting designs. These multimedia formats result particularly effective when the design process needs to integrate a stronger narrative and also when the solution involves use of interactive components.

As the integration of multimedia presentations is explored, it is important to understand their benefits in more detail, in order to develop a final presentation that ensures effective storytelling of design solutions. This understanding allows the creation of a more compelling presentation, capable of reaching broader audiences.

\section{Current practices for product design presentations}

Product Design, as other design disciplines, is a highly visual activity that tends to produce tangible solutions with significant user interaction. Major typologies in product design include: furniture, lighting, tableware, textiles and products. [2]. Product design revolves around a user-centered design process that requires deep user observation and analysis in order to identify unmet user needs. This process includes a sequence that goes from initial observation and research on the user's context, to ideation of potential solutions, creation and testing of two and three-dimensional concepts and mockups, all leading to the final selection and development of a solution [3]. Whenever a design solution is presented, it is important to include all the steps involved in the process so that audiences understand the thinking process and rationale for selection and refinement of the chosen direction.

Traditionally, this process is assembled into a final presentation as a booklet or slideshow, which can be printed or displayed digitally via screen or projection. For public presentations, designers generally use slideshows as visual aid while they give a verbal explanation. The most common format for digital presentation is Microsoft PowerPoint but publishing software programs such as Adobe InDesign have gained strong popularity in recent years. These programs allow for files to be saved as PDF, JPG or other image files, which are easy to distribute and are considered industry standards.

In terms of content, storytelling is an essential element for successful presentations, moving away from simple communication of facts and events and integrating a fluid narrative, more defined personality and emotional qualities that derive from cinematographic styles [4]. A good visual narrative assures that all the components and sequence of a given solution are explained clearly, in a coherent and integrated flow. Storytelling offers benefits that go beyond communication of design, as it also serves as a reflective method of analysis and synthesis, necessary for putting together an effective narrative flow [5]. A good presentation, whether in analog or digital formal, needs to have a strong and coherent storyline, backed up by engaging visuals. In the case of video presentations, effective storytelling is essential as videos tend to run uninterruptedly and 
don't allow for a presenter to step in and explain details that are not clearly understood by viewers. Videos can deliver design solutions in a fluid and fast pace, in contrast to still-frame presentations that depend greatly on the presenter's narrative and ability to engage with the audience.

\section{Use of videos for final presentations}

The process book presentation is based on still images, which can be limiting for explaining situations and product features that exist in a dynamic setting. Although the use of storyboards and visual user scenarios is a common way of communicating sequential information, this is time consuming and doesn't always deliver all the richness and complexity that certain scenarios contain. Videos, on the other hand, are able to capture more contextual information contained within a scene. The use of multimedia components in presentations has shown a steady increase in popularity in recent years, delivering not only final solutions but also being used for educational content [6]. Videos are becoming a popular alternative to presenting design projects and other similar bodies of work. The popularity that multimedia formats receive is also result of the influence that social media has in society.

Digital storytelling allows students to be more reflective on the message that they convey in their presentations. As they edit their videos they are able to analyze images, voiceovers, sound, visual effects, and other elements that communicate their ideas [7]. Throughout this process, students organize their rationale and evaluate their results in a clearer fashion, making videos an excellent vehicle for organizing, communicating and evaluating designs.

\subsection{Different levels of integration}

Developing strong presentation skills is a key component of design education. Professional designers constantly present their ideas to diverse audiences that include engineers, marketers, clients, etc. Students need to learn to present their ideas in a clear way, using universal language that resonates with any audience while also being aware of key terminology for specific disciplines. This is a skill that takes time to develop and the use of video presentations should never replace verbal presentations. Videos can be seen as another communication tool and students should be able to combine different types of methods as they find appropriate. Hybrid presentations are a common model, where students show videos that communicate key elements of their designs, while also providing verbal information that allows them to engage in discussions with their audience. Additionally, videos provide an outlet for the student's personality to come through; creating a piece that conveys the design intent with a personal touch.

\subsection{Use of videos in cross-disciplinary projects}

Design education relies heavily on project-based courses where students apply skills and processes to specific design problems. This is a perfect environment for enabling cross-disciplinary collaboration, where students from different areas work together in addressing complex problems that couldn't be solved by a single discipline. This process of joint conversation and analysis leads to participatory solutions, critical thinking and empowerment [8]. Both students and their future employers appreciate this model as it has proven to be quite effective in real-life business models. The use video presentations can be particularly useful in cross-disciplinary collaborations since videos offer a universal type of output that is easy to understand by broader audiences from across different disciplines and integrates ideas from team members into a joint storytelling piece that reflects the vision of the group [9]. Any person identifies with telling and listening to stories and videos serve as equalizers of the information that needs to be delivered [10]. Also, because of the large amount of information that videos can deliver in a short period of time compared to verbal presentations, cross-disciplinary teams can include more detailed information for each of the areas involved in the project as part of the video or as supplemental information.

\section{Benefits and considerations when using video presentations}

Just like any other method that is introduced into the curriculum, it is important for students to understand the value that videos will have on their communication skills. As part of this understanding, a set of benefits and considerations need to be kept in mind:

\subsection{Benefits of video presentations:}

Increased interest from students: multimedia formats resonate with interests and lifestyles of their generation, making them more excited about creating these types of presentations. Students find videos to be more up-to-date and they tend to share them more widely with peers and in social media channels. This format aligns with the popularity of social media as an effective communication channel for younger generations [11]. 
Multi-layered communication: Videos allow for simultaneous delivery of content, including: images, sounds, narration, audiovisual effects and other symbolic elements. Such richness of content delivers a comprehensive perspective on how design solutions have been conceived and can be implemented in the real world. Videos lend themselves to create scenes that describe product features in-context. Students frequently reenact user scenarios that illustrate information such as human interaction, adaptability to the environment, scale, proportions and usability of the product. This ability is sometimes described as 'video envisionment' where viewers understand how users interact with a solution in context, instead of simply looking at features of a given device [12]. Many of these layers of information would be extremely difficult to convey in a still-frame format.

Narrative over long periods of time: when designing a new device it is important to be aware of its entire lifecycle, from pre-manufacture and manufacture to transportation/distribution, use and disposal [13]. Product features based on whole life cycle thinking often involve multiple geographic locations, user scenarios and lifespans that range long periods of time [14]. Videos offer abundant versatility to recreate and communicate multiple situations and timelines, integrating them into a cohesive story.

Deeper connection: Videos can be quite moving and students often take advantage of this virtue to create video presentations that connect with their audience at strong emotional levels. It is common for students to integrate into videos situations from their personal experiences, giving videos a very personal and authentic feel. The emotional connection achieved by a video not only engages better with viewers but also makes the designs and presentations more memorable.

Access to wider audiences: videos benefit from their dynamic storytelling and ability to communicate large amount of information in relatively short time, making them more compelling and engaging to broader audiences. Given that videos can be easily uploaded to online channels, they are extremely portable and easy to share. The high level of accessibility to video presentations promotes dissemination, conversation and discussion among larger and more diverse audiences, improving the feedback and exposure that students receive from their designs.

\subsection{Considerations for video presentations:}

Along with the benefits just mentioned, it is important to keep in mind the following considerations when creating video presentations:

Time intensive: Videos take significant time to put together and this needs to be taken into account when planning course load. Students should pay attention to steps such as story building, creation of footage, editing and file compression. While programs such as Apple's iMovie simplify this process and makes it more intuitive, students should still plan on working for a couple of weeks on their video. It is also important to mention that making a video does not mean creating a short film. Many great videos come from having dynamic slideshows that combine blocks of text with images, all with music background, giving to audiences the perception of a dynamic format.

Attention to narrative: In order to achieve successful storytelling, it is important that students include the right amount of content in their story line, without leaving critical information out. It is good practice for students to layout storyboards and make sure they are hitting the right points before they begin creating images or shooting scenes. Just as a good design comes from clear objectives and process, a good video needs to have key ideas and messages to be communicated.

Using the right length: If videos are too long, they can easily loose the interest from viewers. Lengths between two and five minutes often provide a good balance of being able to fit enough information to explain the concepts clearly but not long enough to diminish the video's impact. A video shorter than two minutes will suffer from not presenting enough information to understand the design solution, and a video that is too long will end up adding information that is not essential to the design concept and will dilute the message.

Copyrights and privacy: Videos are easy to share with the world and this makes them highly susceptible to issues on copyrights, Internet safety and privacy issues. Guidance on these matters should be made available to students. Social media channels such as YouTube offer good guidelines on copyright and privacy concerns: http://www.youtube.com/yt/copyright/

\section{Examples of Student projects}

The Industrial Design department at Rochester Institute of Technology, in upstate New York, is incorporating the use of video presentations for a wide array of student project presentations. RIT is an 
institution that offers a unique balance between technical disciplines and applied, creative areas like design. Projects that come out from RIT's Industrial Design program often have this balance and are presented to broad audiences inside and outside of the institution. The following projects come from undergraduate and graduate-level courses, moslty setup as inter-disciplinary collaborations.

\subsection{Revolve phone}

Designed by Industrial Design students Chandra Baker, Chris Platt, Da Deng and Jason Schuller, the phone is designed for easy upgrading and disassembly, and for stronger user attachment (see Figure 1). Revolve's video shows a cohesive, engaging narrative that connects user scenarios with specific sustainability strategies. This video shows an intense energy that resonates with younger audiences and key user groups that the team geared the phone towards to.

Link to Video: http://youtu.be/aP6ybB2KNI0

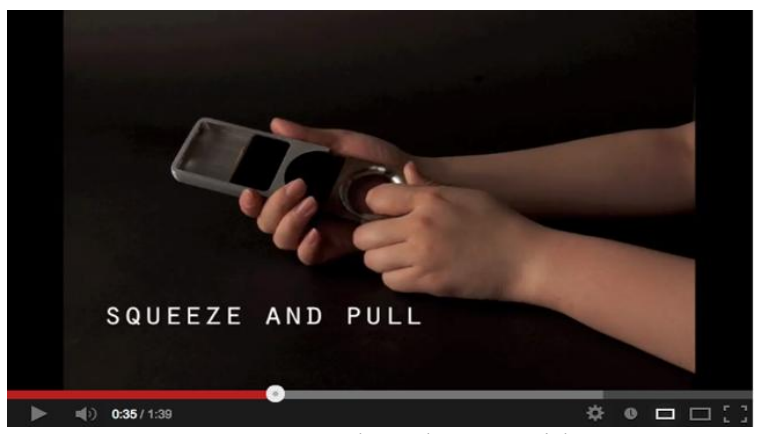

Figure 1. Revolve phone's video.

\subsection{Planters Peanuts Packaging}

Designed by Isaac Alves, Hong Ying Guo, Vicki Julius, Andrew Lakata, Keelyn Nori and Marc Priddy, this updated packaging concept for Planters focuses on an enhanced user experience for people on the go, while highlighting the tradition of the brand in American culture (see Figure 2). For their presentation, the team opted for a hybrid model, where a video was embedded in the middle of a stillframe slideshow. Viewers of this presentation often comment that the video acts as the selling point and makes the design memorable and fun. The presentation as a whole shows good integration of different disciplines and levels of information, as students in industrial design, graphic design and packaging science comprised the team.

Link to presentation: http://goo.gl/dZ6Jr Link to Video: http://youtu.be/mqKoQ8Mvhy0

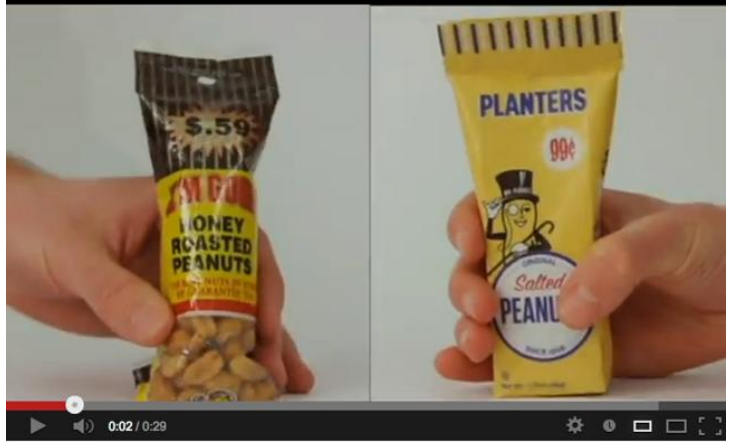

Figure 2. Planters packaging's video

\subsection{FarmLOCAL}

Designed by Industrial Design students Julian Del Campo, Alexander Freeman, Benjamin Hudson and Anton Siekmann, this system allows local farmers to publicize their produce offerings, encouraging consumers and businesses to reach out to vendors and use local goods (see Figure 3). FarmLOCAL's video is successful at transporting the audience to Rochester's farmers market, the precise context where this system will be implemented. Viewers are able to connect with farmers, costumers and market goers in general. This type of connection and engagement with the user's context would be very hard to achieve in a more static format. The video also proved to be a creative outlet for the students at multiple levels, who went as far as acting as customers and also composing the video's music score.

Link to Video: http://youtu.be/zk8EWpFfypc

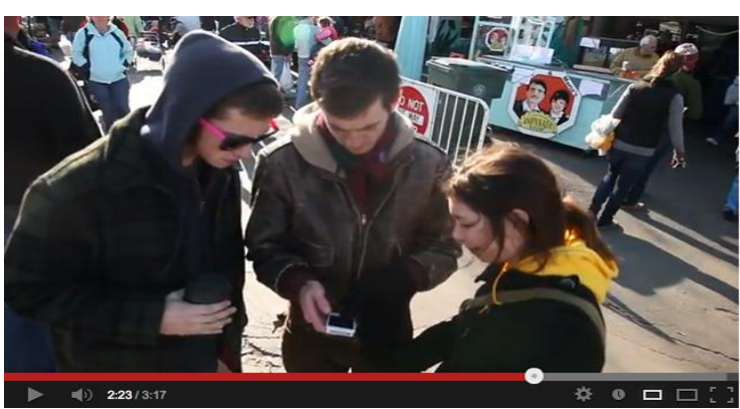

Figure 3. FarmLOCAL online system's video

\subsection{Squat sanitation system}

Designed by Miguel Adames, Amanda Pardee, Briana Stephenson-Vallot, Trevor Thunell, Randall Yarborough and Danwei Ye, students in Industrial Design and Mechanical Engineering, this design offers a sustainable approach to sanitation systems in developing countries (see Figure 4). The video shows a good approach to design for social impact, allowing viewers to understand details and challenges surrounding the context in which the 
solution is planned to exist. Enabling this level of empathy is important for designs what focus on contexts dramatically different from the audience's. The team also focused on producing a video that could be used as a project proposal for seeking external funding.

Link to Video: http://youtu.be/g7q-yzozJH0

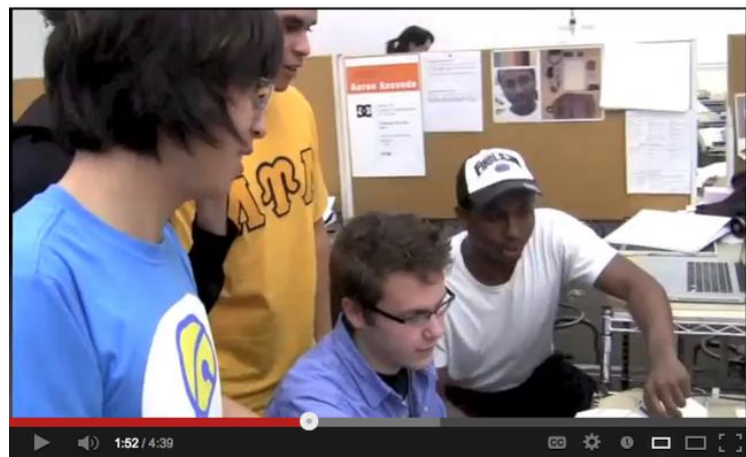

Figure 4: Squat sanitation system's video.

\subsection{LifeGun}

Designed by Kristin McGeorge and Dan Rucker, students in Industrial Design, this device offers a unique method for water purification and filtration for developing countries, based on a portable oneway pumping system that filtrates water, allowing users to then expose the water to the sun's UV rays for further purification (see Figure 5). The video highlights the physical attributes of the design, followed by a design statement that combines technical information with features on the design and considerations for user experience and empowerment, showing yet another type of hybrid presentation that includes verbal delivery of information as part of the video.

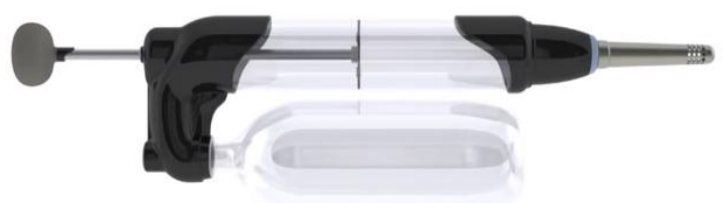

Thelifecun

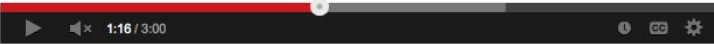

Figure 5. LifeGun video

\section{Conclusions}

Multimedia presentations are becoming extremely popular for sharing design projects and activities. It is important to understand that these types of presentations are not intended to substitute still-frame formats or in-person presentations. There is need and relevance for various formats and designers should become proficient in all of them. In fact, it is quite common to have hybrid presentations that combine both still-frame and multimedia components. This hybrid model is very effective in cases where specific data that needs more time to be explained is combined with components that are presented in a more dynamic flow.

Storytelling is a key component for video presentations, as it assures that the design solution is well communicated. This process makes students reflect about their design and helps them in evaluating their work and synthetize the solutions that they have produced, as they connect with the user needs that they set to design. The learning outcomes from using video presentations transcend the skill set learned in putting them together, also enabling students to be more reflective of their process and results.

Video presentations can be exciting to watch and they are also fun to create, as they offer a fresh medium that resonates with students' interests and lifestyle. The result is a piece that involves skill, creativity and good sense of narrative and storytelling. Video presentations deliver a richer level of communication and storytelling that makes the delivery of a design solution more appealing and effective.

\section{Acknowledgements}

I would like to acknowledge the great work of the students at RIT whose projects are highlighted in this paper and my colleagues Callie Babbitt, Jim Myers, Lorrie Frear, Karen Proctor and Sarah Brownell, with whom I have the fortune of teaching these courses.

\section{References}

[1] Larson, B. (2005) How Designers Think: The Design Process Demystified, Architectural Press, Oxford. p.34.

[2] Hudson, J. (2008) Process, Lawrence King, London, p.7.

[3] Ulrich, K. and Eppinger S. (2011) Product Design and Development, Mc Graw Hill, New York, pp. 13.

[4] Kobre, K. (2012) Videojournalism: Multimedia Storytelling, Focal Press, St. Louis, pp. 5-7. 
[5] Parrish, P. (2006) 'Design as Storytelling', Tech Trends 50 (4), p.72.

[6] Montazemi A. (2006) 'The Effect of Video Presentation in a CBT Environment', Educational Technology \& Society 9(14), pp. 124.

[7] Coventry M. (2008) 'Engaging Gender: Student application of theory through digital storytelling', Arts and Humanities in Higher Education 7, pp. 205.

[8] Trebell, D. (2010) 'Multi-Disciplinary Interaction in Learning Led Design', Design and Technology Education 15(3), p.102.

[9] Lawrence, D. and Thomas J. (1999) 'Social Dynamics of Storytelling: Implications for Story-Base Design', AAAI Technical Report FS-99-01.

[10] Ericksson, T. (1996) 'Design as Storytelling', in Interactions Magazine, AMC Press, New York, vol. 3 (4), p.34.

[11] Hurn, K. (2012) 'The Impact of Social Software in Product Design Higher Education', Design and Technology Education, 17: (2), p. 41.

[12] Buxton B. (2007) Sketching User Experiences: getting the design right and the right design, Morgan Kaufmann, San Francisco, p.349.

[13] Alting, L. and Legarth, J (1995) 'Life Cycle Engineering and Design', in CIRP Annals vol. 44 (2), pp. $571-2$.

[14] Lloyd, P. (2000) 'Storytelling and the Development of Discourse in the Engineering Design Process', Design Studies 21 (4), pp. 366. 